\title{
Syllabic vs segmental perception: On the inability to ignore "irrelevant" stimulus parameters
}

\author{
MICHAEL A. SHAND \\ Phonetics Laboratory, Department of Linguistics \\ University of California, San Diego, La Jolla, California 92093
}

\begin{abstract}
C. C. Wood and R. S. Day have shown that reaction times (RT) to target segments within a syllable are significantly longer when the unattended segment in the syllable (either stop consonant or vowel) is varied randomly than when it is held constant. This has been taken as evidence for the perceptual unity of a stop consonant-vowel syllable. However, this conclusion could be accepted with much more confidence if it could be shown that random variation in unattended segments does not cause an increase in RT if the unattended segments are not in the same syllable as the target segment. The present study, using bisyllabic natural speech stimuli, (1) reconfirms Wood and Day's results, but (2) finds that random variation in unattended nontautosyllabic segments also results in significantly longer RTs than without nontautosyllabic variation. Segmental vs syllabic speech perception thus remains an open question.
\end{abstract}

Numerous studies, using a wide variety of techniques, have shown considerable evidence for the reality of phonemes, phonemic boundaries, and phonetic features (Benson, 1974; Eimas \& Corbit, 1973; Miller \& Nicely, 1955; Studdert-Kennedy \& Shankweiller, 1970). The psychological reality of phonemes (at some level) is not disputed. The question is, rather, whether, in the course of perception, the acoustic signal is decoded into units of phoneme size, with higher analysis referenced to these phonemic units, or whether we first decode the incoming speech signal into units larger than the phoneme (units of roughly syllabic size) and reference further analysis to these "syllabic" units. In the latter case, the identification of phonemic segments would be subsequent to identification of the syllabic units in which the segments occurred. Savin and Bever (1970), employing a reaction time (RT) technique, claim to show precisely this-that syllables are perceived before individual phonemes, and that identification of phonemic segments within a syllable, if and when it occurs, is independent of and subsequent to identification of the syllable in which they occur. They base their conclusion on their finding that RTs to phoneme targets in lists of monosyllabic nonsense words are longer than the RTs to syllable targets. By inference from their arguments, since RTs are longer to the phoneme targets, phonemes are an abstraction from the syllable in which they are contained.

This conclusion is especially interesting in light of a later experiment involving syllable and word identification (Foss \& Swinney, 1973). Foss and

This work was supported by NIH Grant PHSNS-1173501Smith.
Swinney found that RTs to bisyllabic words were faster than RTs to the initial syllable of the word. This was true even if the first syllable of the bisyllabic word was in itself a meaningful word. That is, if the subject is given a list of bisyllabic words and asked to push a button when he hears the word "candy," he will respond faster than when he is told to push the button when he hears "can" (the first syllable in the word "candy"). By Savin and Bever's (1970) reasoning, we should thus conclude that the minimal perceptual unit must be of bisyllabic size. It should be noted, incidentally, that in both of these experiments the RTs were shorter than all units except the phoneme. That is, when the target was a syllable or bisyllabic word, the RT latency was less than the length of the initial syllable. The subject was clearly responding on the basis of information contained in a segment of the acoustic signal smaller than the syllable.

This then leads us to the question of what is being shown by these RT results. Obviously, some factor or factors other than the minimal perceptual unit is/are confounding these results.

Another RT experiment (McNeill \& Lindig, 1973) brings the results of the other RT experiments into better perspective. McNeill and Lindig show that RT is a function of the experimental design-that the "minimum reaction time in such experiments occurs whenever the linguistic level of the target and the search list is the same." Thus, the results of these experiments reflect the influence of "set" or expectation. That is, how easily different-sized units can be brought to consciousness and organized for some response process is dependent on the design of the experiment rather than on the minimal unit of perception. 
In an attempt to clarify the picture, Wood and Day (1975) modified Savin and Bever's (1970) experimental paradigm in a way which they felt would maximize the probability of the subject's perceiving the stimuli segmentally, given that they could perceive segmentally. The experiment utilizes a twochoice speeded classification task with the subject having to identify either the consonant $(/ \mathrm{b} /$ or $/ \mathrm{d} /)$ or the vowel $(/ \mathrm{a} /$ or $/ \partial \mathrm{e} /)$ of a monosyllabic stimulus. In one set of conditions, the irrelevant stimulus dimension is held constant ("control"). In the other set of conditions ("orthogonal"), the irrelevant stimulus dimension is varied randomly (as is the "target" dimension in all conditions). The results show that RTs are consistently faster in the control conditions - that is, it is "easier" to identify which consonant (or vowel) occurred if the unattended vowel (or consonant) is kept constant. Wood and Day reason that if perception is phonemic, or, in any case, segment by segment, the subject should find it beneficial to ignore the irrelevant tautosyllabic segment, and hence the orthogonal condition should not result in an increase in $\mathrm{RT}$. But since RTs are longer for the orthogonal condition, it must be the case (they reason) that subjects are unable to perceive the segments individually.

Wood and Day (1975) assume that variation in parameters which are not within the same minimal perceptual unit do not result in an increase in RT. This assumption is crucial to their argument, yet it was not tested by the experimenters. In order to examine this question, the stimuli must be modified in such a way that there is random variation in an irrelevant dimension which we are reasonably sure is not within the same minimal perceptual unit. For instance, consonant-vowel (CVCV) bisyllabic stimuli could be used (for reasons of economy and simplicity, there is considerable reluctance to postulate minimal perceptual units larger than the syllable). If we find that varying parameters in the initial (always irrelevant) syllable does not affect RTs to the attended components in the second syllable, then we can accept the conclusions of Wood and Day (1975) with much more certainty. If, on the other hand, we find that varying an irrelevant dimension in a different syllable also increases RTs to the target dimension, we will be forced either to the unhappy acceptance of something larger than the syllable as the minimal perceptual unit [analogous to applying Savin and Bever's (1970) arguments to Foss and Swinney's (1973) results] or to conclude that this experimental paradigm, like that of Savin and Bever (1970), is not valid for determining the minimal units for the perception of speech.

\section{METHOD}

\section{Stimuli}

Natural speech syllables spoken by a trained male phonetician were recorded on audiotape. Tokens of the syllables $/ \mathrm{ba}, \mathrm{bre}, \mathrm{da}$, dre, ge, go, ke, ko/ chosen to be as nearly identical as possible in terms of pitch contour were digitized, edited to equal length, amplitude normalized, and stored on LINC tape for on-line presentation. Each stimulus in final form consists of a bisyllabic nonsense word having no transitions between syllables, the first syllable of which is a $300-\mathrm{msec} / \mathrm{ge} /, / \mathrm{go} /, / \mathrm{ke} /$, or $/ \mathrm{ko} /$ and the second of which is a $300-\mathrm{msec} / \mathrm{ba} /, / \mathrm{b} \partial \mathrm{e} / \mathrm{/da} /$, or $/ \mathrm{dae} /$.

\section{Procedure}

The subjects, 12 undergraduates at UCSD, each of whom reported no history of hearing disorders, were each seated comfortably in a sound-attenuated room. The stimuli were presented binaurally by a PDP-12 computer to the subject over matched Telephonics TDH-39 headphones at a comfortable listening level (approximately $80 \mathrm{~dB}$ SPL). The subjects were instructed to respond as rapidly as possible by pressing one of two response buttons (equidistant from the "rest" position) appropriately marked for the relevant targets. Each subject was presented with three conditions of 64 trials each (7-sec ISI) with a replication, yielding a total of 128 responses per subject per condition. Presentation order for conditions was counterbalanced across subjects, using a randomized Latin square design. Prior to the start of each condition, the subject was presented with eight practice trials of the same type as that condition.

The conditions were of three types. In the control conditions, the target segment (either the consonant or the vowel of the final syllable) varied randomly. The initial syllable and the nontarget segment of the second syllable were kept constant for the 64 trials. In the tautosyllabic orthogonal conditions, the initial syllable was kept constant and both segments of the final syllable varied randomly. In the nontautosyllabic orthogonal conditions, the nontarget segment in the second syllable was kept constant while the initial syllable and the target segment of the second syllable varied randomly. In each of these conditions, the subject was instructed to attend only to the target segment. As in Wood and

Table 1

Stimuli

\begin{tabular}{ll}
\hline /ge ba/ & $/$ ke ba/ \\
\hline /ge ba/ & $/$ ke ba/ \\
/ge da/ & $/$ ke da/ \\
/ge da/ & $/$ ke da/ \\
/go ba/ & $/$ ko ba/ \\
/go ba/ & $/$ ko ba/ \\
/go da/ & $/$ ko da/ \\
/go da/ & $/$ ko da/ \\
\hline
\end{tabular}

Table 2

Sample Stimulus Sets by Target Segments and Condition Non-

\begin{tabular}{|c|c|c|c|}
\hline Target Segment & Control & $\begin{array}{c}\text { Tautosyllabic } \\
\text { Orthogonal }\end{array}$ & $\begin{array}{c}\text { tautosyllabic } \\
\text { Orthogonal }\end{array}$ \\
\hline Consonant & $\begin{array}{l}\text { /geba/ } \\
\text { /geda/ }\end{array}$ & $\begin{array}{l}\text { /geba/ } \\
\text { /geba/ } \\
\text { /geda/ } \\
\text { /geda/ }\end{array}$ & $\begin{array}{l}\text { /geba/ } \\
\text { /geda/ } \\
\text { /keba/ } \\
\text { /keda/ } \\
\text { /goba/ } \\
\text { /goda/ } \\
\text { /koba/ } \\
\text { /koda/ }\end{array}$ \\
\hline Vowel & $\begin{array}{l}\text { /geba/ } \\
\text { /geba/ }\end{array}$ & $\begin{array}{l}\text { /geba/ } \\
\text { /geba/ } \\
\text { /geda/ } \\
\text { /geda/ }\end{array}$ & $\begin{array}{l}\text { /geba/ } \\
\text { /geba/ } \\
\text { /keba/ } \\
\text { /keba/ } \\
\text { /goba/ } \\
\text { /goba/ } \\
\text { /koba/ } \\
\text { /koba/ }\end{array}$ \\
\hline
\end{tabular}


Table 3

Mean RT (Milliseconds*) by Target Segment and Condition

\begin{tabular}{cccc}
\hline & \multicolumn{3}{c}{ Condition } \\
\cline { 2 - 4 } Target Segment & Control & $\begin{array}{c}\text { Tautosyllabic } \\
\text { Orthogonal }\end{array}$ & $\begin{array}{c}\text { Non- } \\
\text { tatosyllabic } \\
\text { Orthogonal }\end{array}$ \\
\hline Consonant & 296 & 348 & 332 \\
Vowel & 312 & 346 & 339 \\
\hline
\end{tabular}

*Measured from onset of second syllable

Day (1975), variation in any of the nontarget segments never served as an additional aid in the subject's task. Thus, it was to the subject's benefit to ignore all information except the target segment, and this was brought to the subject's attention. The subjects' responses and RTs were recorded on-line for later analysis.

\section{RESULTS}

The RTs are significantly longer (by analysis of variance) in the tautosyllabic orthogonal conditions for both consonant targets (52-msec difference; $\mathrm{F}=31.22, \mathrm{p}<.001$ for $1,11 \mathrm{df}$ ) and vowel targets (34-msec difference; $F=36.75, p<.001$ for $1,11 \mathrm{df}$ ). This reconfirms the findings of Wood and Day (1975).

However, the RTs in the nontautosyllabic orthogonal conditions are also significantly longer than those in the control conditions for both consonant targets (36-msec difference; $F=36.09, p<.001$ for $1,11 \mathrm{df})$ and vowel targets (27-msec difference; $F=11.62, p<.01$ for $1,11 \mathrm{df}$ ). Error rates in all conditions ranged between $5 \%$ and $10 \%$.

\section{DISCUSSION}

We find that, although Wood and Day's conclusion-that the hearer perceives $\mathrm{CV}$ syllables as a unit-is still a quite plausible one, and one supported by other studies as well (Pisoni \& Tash, 1974), both their results and the results of the present experiment also support another conclusion: that the nature of the human perceptual system is such that some irrelevant dimensions cannot be ignored in processing despite the fact that the subject knows that the particular dimension will in no way aid in the task.
The present experiment clearly shows that this inability to ignore irrelevant stimulus parameters is not dependent on their being within the same minimal perceptual unit. We are led, therefore, to the conclusion that no sound experimental evidence has been brought to bear on the question of the size of the minimal unit in speech perception by either Savin and Bever (1970) or Wood and Day (1975). Although CV syllables may necessarily be processed as a unit, this has yet to be shown satisfactorily, since none of the RT differences found in any of the studies in any way rule out the possibility of segmental perception. Resolution of the size of the minimal unit of perception thus awaits further experimentation.

This experiment then offers a warning: caution must be exercised in designing experiments so that reasonable alternative explanations can be definitely eliminated.

\section{REFERENCES}

Benson, P. J. On some phonetic effects in dichotic listening. Unpublished doctoral dissertation, University of California, San Diego, 1974.

Enmas, P. D., \& Corsit, J. D. Selective adaptation of linguistic feature detectors. Cognitive Psychology, 1973, 4, 99-109.

Foss, D. J., \& Swinney, D. A. On the psychological reality of the phoneme: Perception, identification, and consciousness. Journal of Verbal Learning and Verbal Behavior, 1973, 12, 246-257.

McNeill, D., \& Lindig, K. Perceptual reality of phonemes, syllables, words, and sentences. Journal of Verbal Learning and Verbal Behavior, 1973, 12, 419-430.

Miller, G., \& Nicely, P. E. Analysis of perceptual confusions among some English consonants. Joumal of the Acoustical Society of America, 1955, 27, 338-352.

Pison, D. B., \& TASH, J. Same-different reaction times to consonants, vowels, and syllables. Joumal of the Acoustical Society of America, 1974, 55, 436(A).

SAVin, H., \& Bever, T. G. The non-perceptual reality of the phoneme. Joumal of Verbal Learning and Verbal Behavior, $1970,9,295-302$.

Studdert-Kennedy, M., \& Shankweiller, D. Hemispheric specialization for speech perception. Journal of the Acoustical Society of America, 1970, 48, 579-594.

Wood, C. C., \& DAY, R. S. Failure of selective attention to phonetic segments in consonant-vowel syllables. Perception \& Psychophysics, 1975, 17, 346-350.

(Received for publication March 24, 1976; revision accepted August 13, 1976.) 